\title{
Antenna-Coupled Terahertz Microbolometers with Meander Structures: the Comparison of Titanium and Platinum Thermistors
}

\author{
N. Hiromoto ${ }^{1}$, A. Banerjee ${ }^{2 *}$, E. Durgadevi ${ }^{1}$, H. Satoh ${ }^{2}$, C. Apriono ${ }^{3}$, D. Itoh ${ }^{4}$, E. Bruendermann ${ }^{5}$, \\ E. T. Rahardjo ${ }^{3}$, and H. Inokawa ${ }^{2}$ \\ ${ }^{1}$ Graduate School of Science and Technology, Shizuoka University, Hamamatsu 4328011, Japan \\ ${ }^{2}$ Research Institute of Electronics, Shizuoka University, Hamamatsu 4328011, Japan \\ ${ }^{3}$ Faculty Engineering, Universitas Indonesia, Depok 16424, Indonesia \\ ${ }^{4}$ Faculty Engineering, Shizuoka University, Hamamatsu 4328011, Japan \\ ${ }^{5}$ IBPT, Karlsruhe Institute of Technology, Campus North, D-76344, Germany \\ * Present address: Dept. Electrical and Computer Eng., National University of Singapore, Singapore 119077
}

\begin{abstract}
Uncooled terahertz (THz) detectors with low noise-equivalent power (NEP) are especially important for the application of non-destructive sensing in many fields. Antenna-coupled $\mathrm{THz}$ microbolometers with meander thermistors of titanium (Ti) and Platinum (Pt) are studied to develop room-temperature $\mathrm{THz}$ detectors. The responsivity and NEP of the microbolometer with a meander Ti thermistor are much improved for $1 \mathrm{THz}$ irradiation in comparison with those of the previous one with a straight $\mathrm{Ti}$ thermistor. The $\mathrm{THz}$ performance of the meander $\mathrm{Ti}$ bolometer is much better than the one with a meander Pt thermistor. Those results can be explained by the increase of the thermistor resistance and temperature coefficient of resistance (TCR) through innovating the meander line and also improving the fabrication process based on the electron beam (EB) lithography.
\end{abstract}

\section{INTRODUCTION}

$\mathrm{D}$ EVELOPMENT of $\mathrm{THz}$ detectors with low noise-equivalent power (NEP) is important in wide fields of applications. Room-temperature detectors in frequencies lower than $1 \mathrm{THz}$ are especially indispensable for non-destructive sensing in industry, security, medicine and so on. Moreover, if the $\mathrm{THz}$ direct detectors with the performance enough for passive sensing of room-temperature objects are developed, it will bring the breakthrough to the application of $\mathrm{THz}$ waves.

To solve this issue, we have proposed a room-temperature antenna-coupled microbolometer, comprising of a dipole antenna, a heater connected to it at its center and a thermistor electrically insulated and thermally contacted to them ${ }^{1), 2 \text {, }}$ fabricated the devices on a high-resistivity silicon ( $\mathrm{Si}$ ) wafer by the electron beam (EB) lithography process ${ }^{3)}$ and evaluated them by $\mathrm{THz}$ experiments. We have displayed our detectors have optical performances at $1 \mathrm{THz}$ comparable to the good room-temperature $\mathrm{THz}$ detectors ${ }^{4}$.

In this study, we studied antenna-coupled microbolometers with meander thermistors to obtain higher responsivity and low NEP's by making large resistance of thermistor.

\section{Design, FABRICATION AND EXPERIMENTS}

The configurations of the antenna-coupled microbolometers with meander thermistors are shown in Figs. 1 and 2. The widths and lengths of the meander line of the titanium (Ti) or platinum (Pt) thermistors are $0.1 \mu \mathrm{m}$ and $90 \mu \mathrm{m}$ and $0.2 \mu \mathrm{m}$ and $49 \mu \mathrm{m}$ as shown in the figures.

The $\mathrm{Ti}$ heater is used because $\mathrm{Ti}$ has relatively large

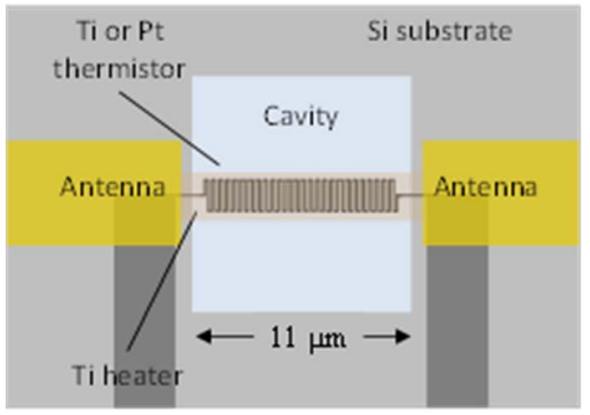

Fig. 1. Configurations of antenna-coupled microbolometers with a 0.1 $\mu \mathrm{m}$-wide meander thermistor of titanium or platinum.

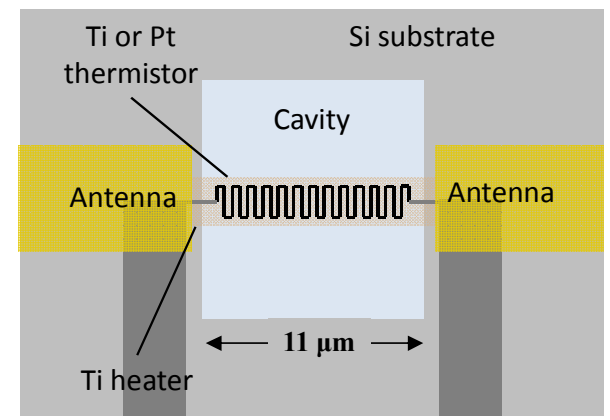

Fig. 2. Configurations of antenna-coupled microbolometers with a 0.2 $\mu \mathrm{m}$-wide meander thermistor of titanium or platinum.

resistivity in all metals and the bulk resistivity of $\mathrm{Ti}$ is 4-time larger than that of Pt. The resistivity of thin lines of Ti was 7.5 time larger and the temperature coefficient of resistance (TCR) was smaller by $1 / 8$ times compared with the bulk value in the previous study, but it brings almost no bad effect to the heater performance. The Pt thermistor is studied besides the $\mathrm{Ti}$ thermistor adopted in the previous study, because $\mathrm{Pt}$ is more stable to oxidation and acid-base reaction in the fabrication process. Insulation layers of $\mathrm{SiN}_{\mathrm{x}}$ for the $\mathrm{Ti}$ and $\mathrm{Pt}$ thermistors are also studied to compare them with the $\mathrm{SiO}_{2}$ insulation layer.

The heater of $\mathrm{Ti}$ is directly connected to the antennas made of gold $(\mathrm{Au})$. The length of the dipole antenna including a $\mathrm{Ti}$ heater is $52 \mu \mathrm{m}$ which fits to resonance length of a half wavelength of $1 \mathrm{THz}$ wave on the Si surface.

The electron beam (EB) lithography process is applied to all the lithographic steps in the device fabrication.

The electrical experiments have been made to measure 


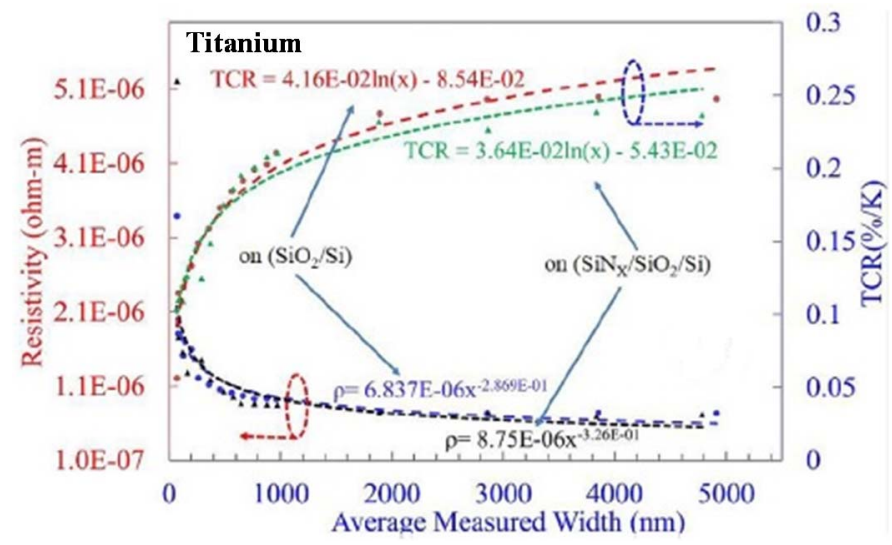

Fig. 3. Resistivity and temperature coefficient of resistance (TCR) as a function of averaged measured width of Ti line fabricated in this study. Results of insulation layers of $\mathrm{SiO}_{2}$ and $\mathrm{SiN}_{\mathrm{x}}$ are also displayed in the figure (Ref. 6).

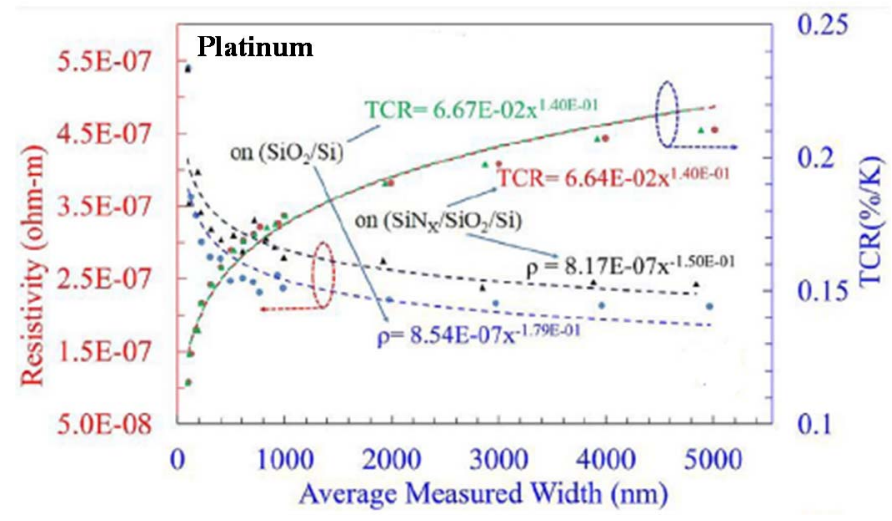

Fig. 4. Resistivity and temperature coefficient of resistance (TCR) as a function of averaged measured width of Pt line fabricated in this study. Results of insulation layers of $\mathrm{SiO}_{2}$ and $\mathrm{SiN}_{\mathrm{x}}$ are also displayed in the figure (Ref. 6).

resistivity, TCR, thermal conductance, noise, and electrical responsivity $^{5}$. In the experiment, alternating current (AC) is supplied to the heater to give modulation of Joule heating, and the modulated output $\mathrm{AC}$ voltage is measured at the thermistor under a bias direct current (DC).

Furthermore, the optical experiments are made to measure responsivity to $\mathrm{THz}$ radiation from a $1 \mathrm{THz}$ Schottky-diode multiplier source and the noise under background radiation at room-temperature by using a low-noise pre-amplifier, and the NEP is evaluated for a thermistor current of $0.1 \mathrm{~mA}$.

\section{RESULTS}

The resistivity and TCR of Ti line are displayed for both the insulation layers of $\mathrm{SiO}_{2}$ and $\mathrm{SiN}_{\mathrm{x}}$ as functions of the line width in Fig. 3. The results show that the resistivity increases and the TCR decreases as the line width decreases, and also the differences in the resistance and TCR are small between the two insulation layers.

The increase in resistivity is about 4 times and the decrease in TCR is about $1 / 3$ times between $100 \mathrm{~nm}$ wide line and bulk material value, which shows the deterioration is eased compared with the previous study. These good results are produced by the improvement in the fabrication process.

Figure 4 displays the resistivity and TCR of Pt line as functions of the line width for insulation layers of $\mathrm{SiO}_{2}$ and
$\mathrm{SiN}_{\mathrm{x}}$. This figure shows the Pt line has also large dependence in both the resistivity and TCR on the line width and the small difference between the insulation layers.

The ratio of resistivity between $\mathrm{Pt}$ and Ti lines is about $1 / 6$ in $100-200 \mathrm{~nm}$ width, which is smaller than $1 / 4$ of the ratio between Pt and Ti bulks. This means that the electric property in the Pt line is less suffered by the fabrication process compared with the Ti line. The TCR of the Pt line is, however, almost the same with that of the Ti line in 100-200 nm width, which is similar with the almost common TCR of the bulk metals of about $0.3 \%$.

The resistances of Ti and Pt thermistors of $0.1 \mu \mathrm{m}$ wide and $90 \mu \mathrm{m}$ long meander lines in the $\mathrm{THz}$ antenna-coupled microbolometers are $16 \mathrm{k} \Omega$ and $4 \mathrm{k} \Omega$ respectively. The THz responsivity of Ti's is 36 time higher than that of Pt's. The noise of the Ti's was 4 time larger than the Pt's. If the $\mathrm{THz}$ radiation power within the area equal to square of the wavelength on the Si surface $(119 \mu \mathrm{m}$ for $1 \mathrm{THz})$ is absorbed, the NEP of the antenna-coupled microbolometers with $\mathrm{Ti}$ and Pt meander thermistors are $1.8 \times 10^{-10} \mathrm{~W} / \mathrm{Hz}^{1 / 2}$ and $1.5 \times 10^{-9}$ $\mathrm{W} / \mathrm{Hz}^{1 / 2}$ respectively, which shows the Ti's is 12 time better than the Pt's. It is improved by 2.5 times compared with the previous study ${ }^{4}$. These results can be explained by the increase in the resistance and TCR of Ti lines of $100 \mathrm{~nm}$ order $^{5), 6)}$.

The $\mathrm{THz}$ responsivity of the antenna-coupled microbolometer with Ti thermistor is consistent with the electrical measurement. It is also consistent with the theoretical analysis using the effective area of antenna deduced by the electromagnetic simulation and the area in which the antenna absorbs the $\mathrm{THz}$ irradiation.

\section{SUMMARY}

We have studied a $\mathrm{THz}$ antenna-coupled microbolometer with a meander-line thermistor having large resistance and TCR to realize a room-temperature THz detector with low NEP. The new detector with a meander Ti thermistor has shown NEP improved by 2.5 times compared with the previous detector with a straight thermistor.

\section{ACKNOWLEDGEMENT}

This work was supported by the JSPS KAKENHI Grant Number JP15H03990. Grant-in-Aid for Scientific Research (B), the JST Industry-Academia Collaborative R\&D Program (Collaborative Research Based on Industrial Demand), "Terahertz-wave: Towards Innovative Development of Terahertz-wave Technologies and Applications", and the Cooperative Research Project of the Research Center for Biomedical Engineering with Research Institute of Electronics, Shizuoka University.

\section{REFERENCES}

[1]. N. Hiromoto, 9th Takayanagi Kenjiro Mem. Symp. 4th Int. Symp. Nanovision Science, p. 124 (2007).

[2]. M. Aoki, M. Takeda, and N. Hiromoto, Proc. Int. Conf. Global Research Education (Inter-Academia), p. 65 (2012).

[3]. A. Tiwari, H. Satoh, M. Aoki, M. Takeda, N. Hiromoto, and H. Inokawa, Int. J. Chem. Tech. Res. 7, 1019 (2014-2015).

[4]. N. Hiromoto, A. Tiwari, M. Aoki, H. Satoh, M. Takeda, and H. Inokawa, 39th Int. Conf. Infrared, Millimeter, and $\mathrm{THz}$ Waves (IRMMW-THz), R3/A-27.6 (2014).

[5]. A. Banerjee, H. Satoh, A. Tiwari, Catur A., Eko T. R., N. Hiromoto and H. Inokawa, Jpn. J. Appl. Phys. 56, 04CC07 (2017).

[6] A. Banerjee, H. Satoh, Y. Sharma, N. Hiromoto, and H. Inokawa, Sensors and Actuators A: Physical, 273, 49-57 (2018). 